\title{
System of Diagnostics of the High-Power Accelerator during Formation of Pulsed Bremsstrahlung
}

\author{
A.M. Chlenov, D.M. Ivaschenko*, N.G. Mordasov and V.D. Shiyan \\ Research Institute of Scientific Instruments, Lytkarino, Russia
}

\begin{abstract}
System of diagnostics of the high-power electron accelerator based on undisturbing methods and measuring tools of electron beam characteristics as well as amplitude-time characteristics of the pulse forming system is presented. The analysis of amplitude-time characteristics reproducibility of the electromagnetic pulse formation system, electron beam spectral characteristics and properties of bremsstrahlung in the position of test specimen under given modes of operation of the accelerator performs using developed software. Possibilities of complex diagnostics of the power accelerator electron beams acceleration regime and accelerator units are illustrated by analysis of the accelerating tube parameters.
\end{abstract}

PACS numbers: 41.75.Fr, 41.75.Lx, 41.85.Ew, 41.85.Qg

\section{Introduction}

High-power direct acting pulse electron accelerator came into wide use in the modern science and engineering. Nevertheless, amplitude characteristics of electron and braking radiation on the output of many accelerators characterize low values of the reproducibility from pulse to pulse, which is reflected on the quality and efficiency of the radiation technology. It is required for the optimal solving of this problem to combine the system of diagnostics of accelerating path main components and monitoring of bremsstrahlung parameters.

\section{Measuring technique}

The suggested solution consists in establishing a link between electron beam parameters at the input of the converting target and bremsstrahlung parameters after the converting target in the known geometry of measurements based on use of "transparent" methods and measurers. In this case the parameters of the high-power electron beam are input characteristics of the converting target as a converter, and the parameters of the bremsstrahlung in a chosen medium and geometry are output characteristics of this converter. It is desired to determine static transfer characteristics of the converter at the given conditions of transportation of the high-power electron beam [4].

For this purpose a graduation of measurers carries out in the conditions and operating mode of this accelerator, that allows us to determine static transfer characteristic of the converting target through the measuring of current

* corresponding author; e-mail: dmi@lytkarino.net
$I(t)$, dose rate $P_{\gamma}(t)$ and energy release in the converting target $\mathrm{W}[5]$.

The results of calibration of the measurers and determination on their basis spectral-energetic characteristics of the electron beam are a core of the system of diagnostics of the mode of accelerator operation and the real-time dosimetric accompaniment of radiation treatments. In so doing the approaches to the accelerator converting target as a measuring converter suffered fundamental changes. As the conducted researches showed up, in the most part of radiation practices the accelerator converting target fulfils the function of an anode, which in view of showing effects complicates the determination of the accelerated electrons beam current. The affected beam current measuring effects show on the all pulse forming process. So as to measure the current in the vacuum tube accelerating gap a device was realized that forms a basis for the accelerator converting part [6].

After calibration the converter of bremsstrahlung dose rate can be placed on different distances from the second layer of the target axially beam transportation, depending on the test specimen isodose radiation plane with predicted characteristics of the affecting factor. Dependence between the electron beam current $I$, electron energy $E$ and bremsstrahlung dose rate $P_{\gamma}$ in the test specimen radiation plane was determined relative to these relations by calibration, expressed in the analytical form as

$$
\frac{P_{\gamma}(x)}{K(x) I}=C E^{n}, \quad n=1 /\left(a+b E+c E^{2}+\ldots\right),
$$

where: $P_{\gamma}(x)$ - bremsstrahlung dose rate in the test specimen radiation plane at a distance $x$ from the converting target; $I, E$ - electron current and energy, respectively; $K(x)$ - attenuation coefficient of the bremsstrahlung dose rate in relation to the calibration 
plane; $C, a, b, c$ - power series coefficients, determined by results of the measuring system calibration.

Value $K(x)$ is determined from the relative character of the bremsstrahlung dose rate attenuation axially radiation of the accelerator.

A related diagnostic system was made for the high-power direct acting electron accelerator UIN-10.

Electrical analogs of the signals $U_{l}, I_{l}, I_{d}$ and $P_{\gamma}$, presenting voltage and current on the input of the coaxial vacuum line, current in the vacuum diode and bremsstrahlung dose rate at a chosen point of the field along the electron beam transportation axis, respectively, are shown in Fig. 1.

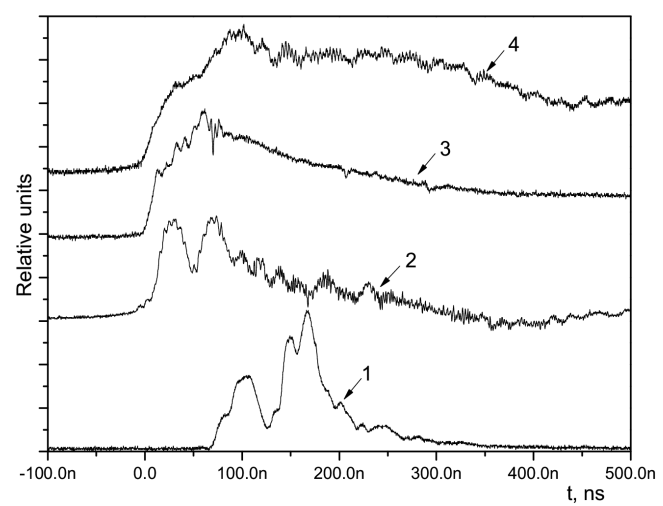

Fig. 1. Signals from measuring channels: $1-P_{\gamma}$, $2-U_{c v l}, 3-I_{d}, 4-I_{c v l}$.

After their treatment by the methods $[4,8]$ information about the effective electron energy in a pulse in the converting target plane was obtained (Fig. 2), on the basis of it spectral-energetic characteristics of the bremsstrahlung and electron beam was determined.

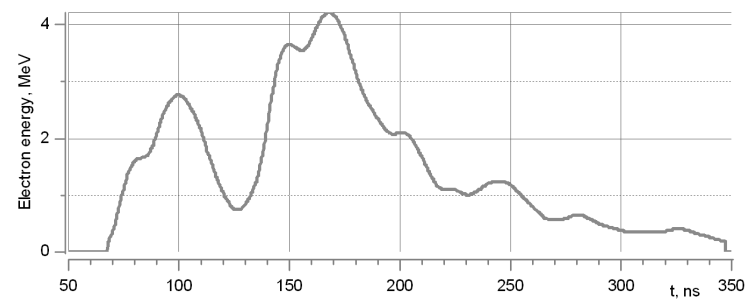

Fig. 2. Distribution of electron effective energy in a pulse.

\section{Results and discussion}

Using the obtained experimental results (Figs. 1, 2), by application of the system diagnostics software [8], it is possible to estimate distribution and losses of energy $W(t)$ in the circuit coaxial vacuum line - vacuum diode in a pulse (Fig. 3), in a transpose time mode, expressed as:

$$
W(t)=U_{c v l}(t) I_{c v l}(t)-U_{d}(t) I_{d}(t),
$$

where $U_{c v l}$ and $I_{c v l}$ are voltage and current at the beginning of the coaxial vacuum line, $U_{d}$ and $I_{d}$ are voltage and current at the vacuum diode.

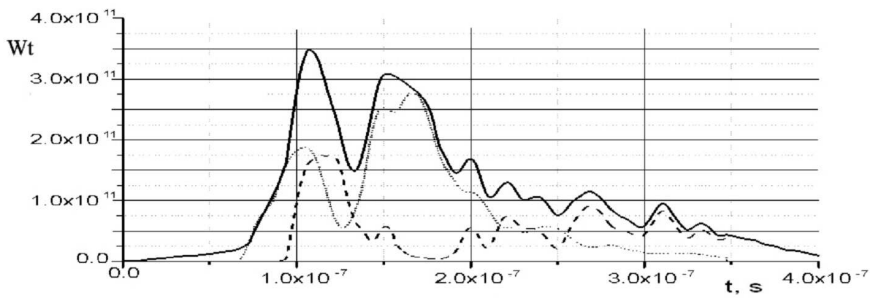

Fig. 3. Distribution of electromagnetic pulse energy in the accelerating tube: solid line - on the input of the accelerating tube, dotted line - on the vacuum diode, dashed line - energy losses in the coaxial vacuum line.

In this equation the first term presents the time response of the pulse energy at the input of the coaxial vacuum line and the second term is the energy of the electron beam in the vacuum diode.

From the experiment follows (Fig. 3) that the maximum of the energy losses with duration less than $30 \mathrm{~ns}$ in the beginning of pulse takes place in a settling time of the magnetic self-insulation in the line.

The theory holds [9] that under the steady state of the magnetic self-insulation in the line the energy losses in the coaxial vacuum line are rather small (less than $15 \%$ from the pulse energy). In the experiment the related losses in the coaxial vacuum line, determined within boundaries of the electromagnetic pulse time interval from $3 \times 10^{-7}$ to $2.5 \times 10^{-7} \mathrm{~s}$ (Fig. 3 ), substantiate these expectations.

The settling time of the magnetic self-insulation mode in the coaxial vacuum line is determined by its parameters and characteristics of the leading edge of the electromagnetic pulse at the line input. Time variation of the accelerating tube and vacuum diode input impedances in the pulse are shown in Fig. 4.

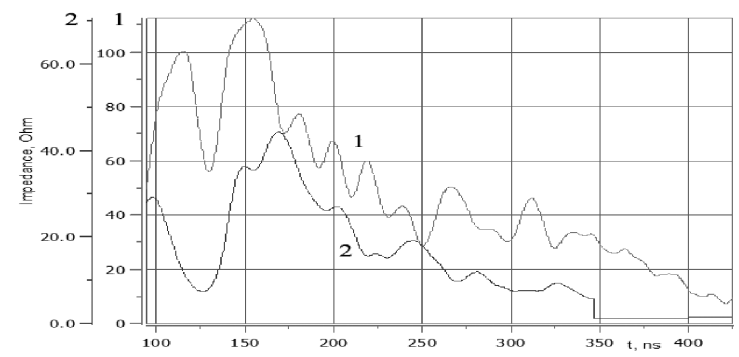

Fig. 4. Impedance of the acceleration tube (2) and vacuum diode (1).

In this manner, using as a basis for the building of diagnostic system the determination of the electron beam energetic characteristics, with the program methods it 
may be defined not only the reproducibility of the electron beam parameters relative to the reference operating mode of the accelerator, but also the association of them with operating conditions of high-voltage units of the accelerating line.

A distinguishing characteristic of the observed in the experiment steady-state magnetic self-insulation conditions is an exceeding of the output current in the coaxial vacuum line $I_{d}$ in comparison to the input current $I_{l}$ (Fig. 1), which determines a degree of unmatched load and voltage drop on it. As illustrated in Fig. 3, the energy losses in establishment of the magnetic self-insulation in the coaxial vacuum line are comparable with energy in the vacuum diode on the initial time interval of the electromagnetic pulse. They comprise less than $30 \%$ of the intermediate inductive storage energy.

\section{Conclusions}

The automated system of diagnostics of the high-power electron accelerator allows us determining not only spectral-energetic characteristics of bremsstrahlung and electron beam, but characteristics of units in the pulse forming system and their effect on reproducibility of the electron beam characteristics as well.

The energy losses in the coaxial vacuum line on the time interval of the electromagnetic pulse with steady-state magnetic self-insulation are close to the designed.

The energy losses during the settling time of the magnetic self-insulation in the coaxial vacuum line have a determining effect on the formation of the pulse-rise time of the electromagnetic pulse on the vacuum diode and the amplitude characteristics of the electron beam and bremsstrahlung.
Determined by means of the diagnostic system time responses of the coaxial vacuum line and vacuum diode impedance allow to estimate methods of matching the load with the pulse voltage generator and to optimize quantitative and qualitative performances of the high-power electron accelerators in radiation technologies.

\section{References}

[1] N.G. Mordasov, J. Technical Physics 73, 107 (2003).

[2] N.I. Zavada, P.L. Komarov, V.A. Tsukerman, E.N. Donskoy, Characteristic of bremsstrahlung and their connection with parameters of high current electron beams, Ed. Sarov, VNIIEF, Russia 1996, p. 490 (in Russian).

[3] S.Y. Sokovnin, Prib. Tekh. Eksp. 4, 125 (1992).

[4] N.G. Mordasov, V.N. Ulimov, V.A. Bryxin, V.D. Shiyan, Prib. Tekh. Eksp. 2, 1 (2005).

[5] D.M. Ivaschenko, N.G. Mordasov, A.M. Chlenov, Proc. 15th International Conference on High-Power Particle Beams, St. Petersburg, Russia, 2004, p. 581.

[6] N.G. Mordasov, A.M. Chlenov, D.M. Ivaschenko, Patent for invention 2281532, G01 T1/36, 2006.

[7] V.A. Bryxin, A.M. Chlenov, A.A. Fedorov, D.M. Ivaschenko, V. Kamensky, V.V. Kochergin, A.P. Metelev, I.N. Mikulin, V.P. Vyskubov, UIN-10 Proc. 15th International Conference on High-Power Particle Beams, St. Petersburg, Russia, 2004.

[8] D.M. Ivaschenko, N.G. Mordasov, A.M. Chlenov, V.D. Shiyan, J. Technical Physics 77, 66 (2007).

[9] L.E. Aranchuk, E.I. Baranchikov, A.V. Gordeev, J. Technical Physics 59, 142 (1989). 